\title{
Additive effects of green LED light and cerium oxide nanoparticles on the planarian's regeneration
}

\author{
A. M. Ermakov ${ }^{1}$, K. A. Kamenskikh ${ }^{1}$, A. L. Popov ${ }^{1}$, \\ O. N. Ermakova ${ }^{1}$, V. A. Afanasyeva ${ }^{1}$, V. K. Ivanov ${ }^{2}$ \\ ${ }^{1}$ Institute of Theoretical and Experimental Biophysics of the Russian Academy of Sciences, \\ Institutskaya str., 3, Pushchino, 142290, Russia \\ ${ }^{2}$ Kurnakov Institute of General and Inorganic Chemistry of the Russian Academy of Sciences, \\ Leninskiy prosp., 31, Moscow, 119991, Russia \\ ao_ermakovy@rambler.ru, kristina.kamensk@mail.ru, antonpopovleonid@gmail.com, \\ beoluchi@yandex.ru,va_vera_afanaseva@mail.ru,van@igic.ras.ru
}

\begin{abstract}
Nanotechnology makes it possible to design advanced materials being able to effectively modulate radiation effects on a cell, depending on the radiation intensity, wavelength, and type. Today, one of the most promising UV and X-ray protective biomaterials is nanocrystalline cerium oxide $\left(\mathrm{CeO}_{2}\right)$, which has a unique redox activity due to its surface reducibility. Meanwhile, the modulating properties of $\mathrm{CeO}_{2}$ nanoparticles when the cells are exposed to visible light remain completely unexplored. Here, we analyzed the impact of $\mathrm{CeO}_{2}$ nanoparticles on the process of planarian regeneration after exposure to low-intensity green LED light. It was found that a one-time exposure (10 or 25 min) of regenerating planarians with low-intensity green light reduced head blastema growth rate by up to $20 \%$. At the same time, the preliminary treatment of planaria with $\mathrm{CeO}_{2}$ nanoparticles in nanomolar concentrations $\left(10^{-11} \mathrm{M}\right)$ ensures the restoration of the neoblasts activity and a significant acceleration of blastema regeneration. Thus, we have firstly demonstrated that the planarian regeneration process can be promoted by cerium oxide nanoparticles even under adverse action of low-intensity green light radiation.
\end{abstract}

Keywords: cerium oxide nanoparticles, green LED, planarian regeneration.

Received: 29 January 2021

Revised: 22 March 2020

\section{Introduction}

The unique antioxidant activity of cerium oxide nanoparticles is based on their ability to participate easily in various redox processes involving reactive oxygen species (ROS) under physiological conditions [1,2]. According to numerous quantum mechanical calculations, the energy of oxygen vacancies formation on the cerium oxide nanoparticle surface decreases with decreasing particle size [3]. Consequently, ultra-small nanoparticles with a high degree of monodispersity are considered to be the most biologically active.

The development of new experimental test models for screening the toxicity and biological activity of new substances and compounds, including nanomaterials, is still an urgent task. Freshwater flatworms - planaria, which have a unique ability to regenerate due to neoblast stem cells, can serve as a good model for such testing [4]. Planarian neoblasts are the only totipotent stem cells in animals that differentiate into all types of cells, including reproductive cells, in an adult organism. The number of neoblasts in the body of planarians is $20-30 \%$ of the total number of cells [5]. These cells allow for unlimited regeneration of planarians both in the event of damage and during the ongoing process of self-renewal of tissues and organs [6]. Moreover, regeneration is even possible from very small fragments where at least one stem cell has been preserved [7]. The specific features of planarians made them a classical experimental model for research of stem cell proliferation and differentiation in vivo, regeneration of differentiated tissue, cell cycle, and aging [8].

We have previously shown that cerium oxide and cerium fluoride nanoparticles are able to accelerate blastema growth due to redox activated expression of genes associated with wound healing [9]. A significant increase in the number of neoblasts in animals treated with $\mathrm{CeO}_{2}$ or $\mathrm{CeF}_{3}$ nanoparticles was observed 3 days after decapitation of the tested planaria. It is worth noting that cerium fluoride nanoparticles showed a higher regenerating ability and efficiency, which is probably associated with the presence of only trivalent cerium $\left(\mathrm{Ce}^{3+}\right)$. It was also shown earlier that cerium oxide nanoparticles provide anti-inflammatory, antioxidant and wound-healing effects in living tissue [10], which makes it possible to develop new cerium-based regenerative preparations [11]. Additionally, cerium oxide nanoparticles exhibit very special antioxidant properties, acting as a scavenger for reactive oxygen species (ROS). It was previously shown that $\mathrm{CeO}_{2}$ nanoparticles act as inorganic nanozyme which has a mimetic activity 
of various endogenous enzymes, such as catalase, glutathione peroxidase, SOD, phosphatase, and a number of other enzymes $[12,13]$. Due to its antioxidant properties, nano- $\mathrm{CeO}_{2}$ is an efficient UV protector capable of protecting mammalian cells from UVA $[14,15]$ and UVB $[16,17]$ radiation. The UV-protective role of $\mathrm{CeO}_{2}$ nanoparticles is not only associated with a decrease in the production of intracellular ROS, pro-inflammatory cytokines, $\beta$-galactosidase activity and suppression of the phosphorylation of $\mathrm{N}$-terminal c-Jun kinases (JNK), but also with the activation of the DNA repair system induced by UV rays. In this case, electromagnetic radiation is capable of damaging biological objects through various mechanisms, including direct damage to DNA or indirect, through the formation of ROS.

Even low doses of UV radiation can cause DNA damage with subsequent activation of the repair system. DNA damage causes activation of signaling protein kinases ATM and ATR [18]. Previously, it was shown that $\mathrm{CeO}_{2}$ nanoparticles are capable of accelerating the repair of damaged DNA fragments, increasing the survival of cells after high-dose irradiation [19]. The antioxidant system of a cell is also activated through increased levels of antioxidant enzymes, e.g. SOD, catalase, glutathione peroxidase, etc. The possibility for activating different cell defense mechanisms depends on the moment of the onset of an unfavorable factor and its duration. The protection against primary (direct) damage is possible only immediately at the moment of irradiation. Protection against secondary damage is determined by the lifetime of the ROS formed during irradiation. Thus, $\mathrm{CeO}_{2}$ nanoparticles are able to reduce radiation-induced cell damage after exposure to high doses of X-ray irradiation [20-22], reducing the level of intracellular ROS and the number of apoptotic cells, as well as the number of cytogenetic lesions. It has also been shown that the radioprotective efficiency of $\mathrm{CeO}_{2}$ nanoparticles varies greatly depending on the radiation energy. For example, Briggs et al. [21] found a significant decrease in the efficiency of radioprotection of cells exposed to $\mathrm{CeO}_{2}$ nanoparticles and irradiated with $10 \mathrm{MV}$ or $150 \mathrm{kVp} \mathrm{X}$-rays. When exposed to low energy irradiation, $\mathrm{CeO}_{2}$ nanoparticles function as a radiosensitizer, which is associated with an increase in the production of Auger electrons with high linear energy transfer. This study highlights an interesting phenomenon which should be considered when $\mathrm{CeO}_{2}$ nanoparticles are used as radiation shielding drugs in radiation therapy. Thus, it can be concluded that the protective efficiency of $\mathrm{CeO}_{2}$ under irradiation conditions is determined not only by the conditions of its synthesis or stabilizer used, but can also vary greatly depending on the type of radiation and its energy.

Here, we investigated the effect of citrate-stabilized ultra-small $\mathrm{CeO}_{2}$ nanoparticles on the rate of regeneration of planarians upon the irradiation with green light $\left(\lambda_{\max }=520 \mathrm{~nm}\right)$, and analyzed the factors that determine the possibility of increasing the efficiency of such protection.

\section{Materials and methods}

\subsection{Preparation of cerium oxide nanoparticles and their characterization}

To prepare cerium oxide nanoparticles sol, $0.24 \mathrm{~g}$ of citric acid was dissolved in $25 \mathrm{ml}$ of $0.05 \mathrm{M}$ aqueous solution of cerium nitrate (III), rapidly poured into $100 \mathrm{ml}$ of $3 \mathrm{M}$ ammonia solution with stirring and allowed to stand for $2 \mathrm{~h}$. According to the transmission electron microscopy data the obtained sol consisted of slightly aggregated isotropic $\mathrm{CeO}_{2}$ nanoparticles of $2.0-2.5 \mathrm{~nm}$ size. The $\mathrm{pH}$ of cerium oxide sol was in the range of 7.2-7.4. The size and shape of $\mathrm{CeO}_{2}$ nanoparticles were determined by transmission electron microscopy (TEM), on a Leo912 AB Omega electron microscope (accelerating voltage $100 \mathrm{kV}$ ). The hydrodynamic size and zeta-potential of $\mathrm{CeO}_{2}$ nanoparticles were measured using a Malvern Zetasizer Nano ZS Analyzer.

\subsection{Green irradiation of planarians}

The LED matrix (LED) with a wavelength of $\lambda_{\max }=520 \mathrm{~nm}$ was used as a light source. The spectral characteristics of the LEDs were analyzed using a set of spectral equipment based on the MDR-41 monochromator (OKBSPEKTR, Russia) (see Fig. 1A). The luminous flux density was measured using a CMR 3 pyranometer (Kipp@Zonen, the Netherlands). The LED power was set at $1.46 \mathrm{~W} / \mathrm{m}^{2}$ varying the distance from the detector to the source.

Before the experiments, citrate-stabilized ceria sol was mixed with a pond water to obtain a colloid solution containing $10^{-11} \mathrm{M} \mathrm{CeO}_{2}$. Planarians (30 animals in each group) were decapitated with a scalpel and placed in a Petri dish (diameter of $6 \mathrm{~cm}$ ) filled with ceria-containing colloid solution $(2 \mathrm{~mm}$ deep). The petri dish was placed on a flat glass plate, the light source was fixed under the glass plate. The emission spectrum of the light source is shown in Fig. 1A (insert). The whole scheme of experiment showed in Fig. 1B. After $10 \mathrm{~min}$, the green LED light source was switched on, and the irradiation was conducted for 10 or $25 \mathrm{~min}$ (the radiation doses of 70.1 and $175.2 \mathrm{~mJ} / \mathrm{cm}^{2}$, respectively). After irradiation, the planarians were left in $\mathrm{CeO}_{2}$ colloid solution for 72,96 or $120 \mathrm{~h}$. After such incubation, morphometric analysis was performed. 

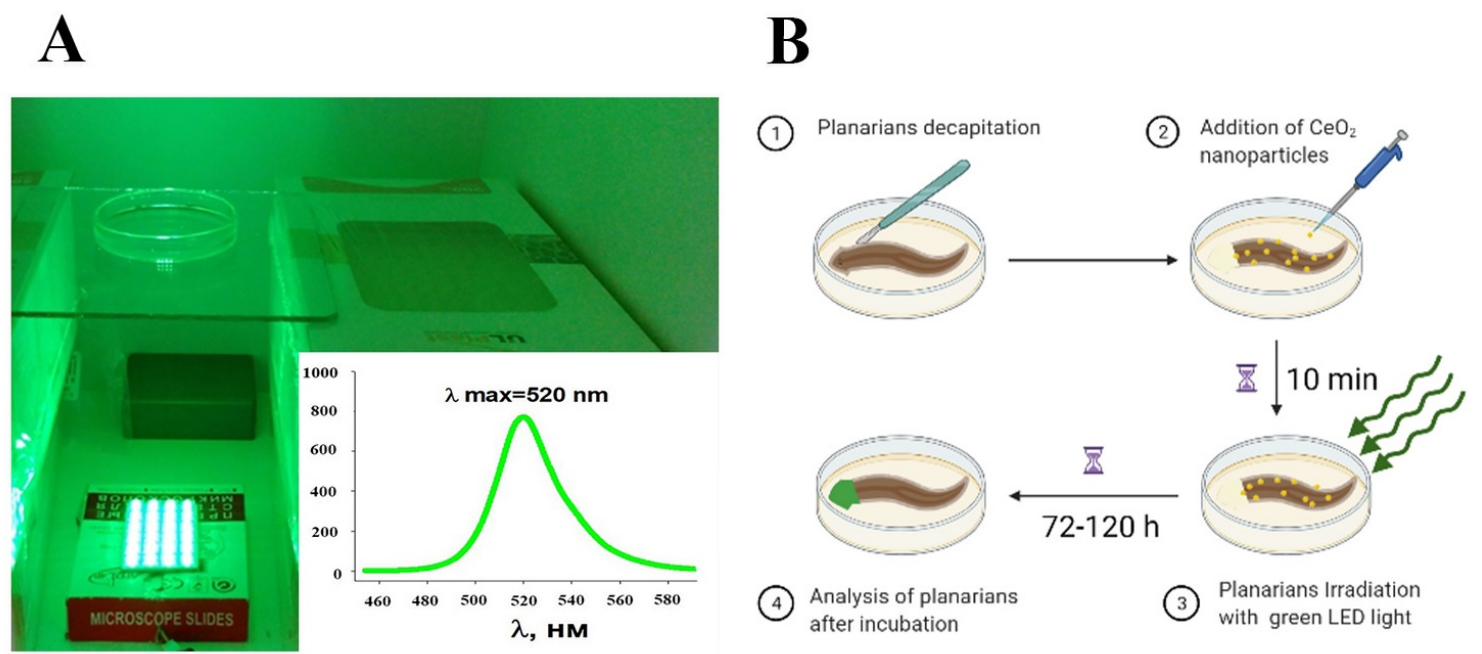

FIG. 1. The spectral characteristics of the LED matrix used for light irradiation of regenerating planarians (A) and the scheme of experiment (B)

\subsection{The morphometry of regenerating planarians}

The growth dynamics of the planarian blastema (a new regenerating head tissue) was evaluated by intravital morphometry [23]. For this purpose, planarians were photographed on the 3rd, 4th and 5th regeneration days using a MRc camera (Carl Zeiss, Germany) mounted on the binocular microscope MBS-10 eyepiece. In the obtained photos, we determined the total area of the animal's body (S) and the area of the blastema (s) using the Plana 4.4 software. The regeneration index $\mathrm{R}=\mathrm{s} / \mathrm{S}$ served as a quantitative growth criterion. Each of the measured $\mathrm{R}$ values is the result of averaging the measurements of $30+$ animals. The regeneration index changes in the experiment $\left(\mathrm{R}_{E}\right)$ compared to the control $\left(\mathrm{R}_{C}\right)$ was determined by the formula:

$$
\Delta R=\frac{\left(R_{E}-R_{C}\right) \pm\left(\delta_{E}+\delta_{C}\right)}{R_{C}} 100 \%
$$

where $\Delta R$ is the difference $(\%)$ between the $R_{E}$ and $R_{C}$ values, $\delta_{(E, C)}$ are the standard errors of measurements for the control and experimental groups. The standard error $(\Delta R)$ did not exceed $6,5 \%$. The control animals were not treated with light and/or nanoceria.

\subsection{Statistical processing}

The data obtained were treated statistically by the Sigma-Plot 9.11 software (Systat Software Inc., Germany) using the one-way ANOVA method.

\subsection{Ethical standards}

All the procedures involving animals were performed in accordance with the ethical standards of the institution at which the studies were conducted (ITEB RAS).

\section{Results and discussion}

The synthesis of $\mathrm{CeO}_{2}$ nanoparticles was carried out according to the previously reported protocol [24]. Transmission electron microscopy data confirm the ultra-small size of cerium oxide nanoparticles and their high degree of crystallinity (Fig. 2A). The hydrodynamic radius of the nanoparticles upon dilution in bidistilled water was 4-7 nm (Fig. 2B). The obtained cerium(IV) oxide nanoparticles possessed catalase-like properties, as we showed earlier by the polarographic method [25], which confirms their antioxidant activity.

As Fig. 3 shows, cerium oxide nanoparticles at a concentration of $10^{-11} \mathrm{M}$ stimulated the growth rate of the planarian head blastema. Thus, after $72 \mathrm{~h}$ of regeneration, the size of head blastema was on average $38.7 \%$ larger than in the control group. In the subsequent observation periods of head growth after decapitation (96 and $120 \mathrm{~h}$ ), the stimulation effect of ceria on regeneration remained, while the average values of the blastema area increment somewhat decreased, $27.2 \%$ and $20.8 \%$, respectively, which agrees well with our recently reported data [9]. 

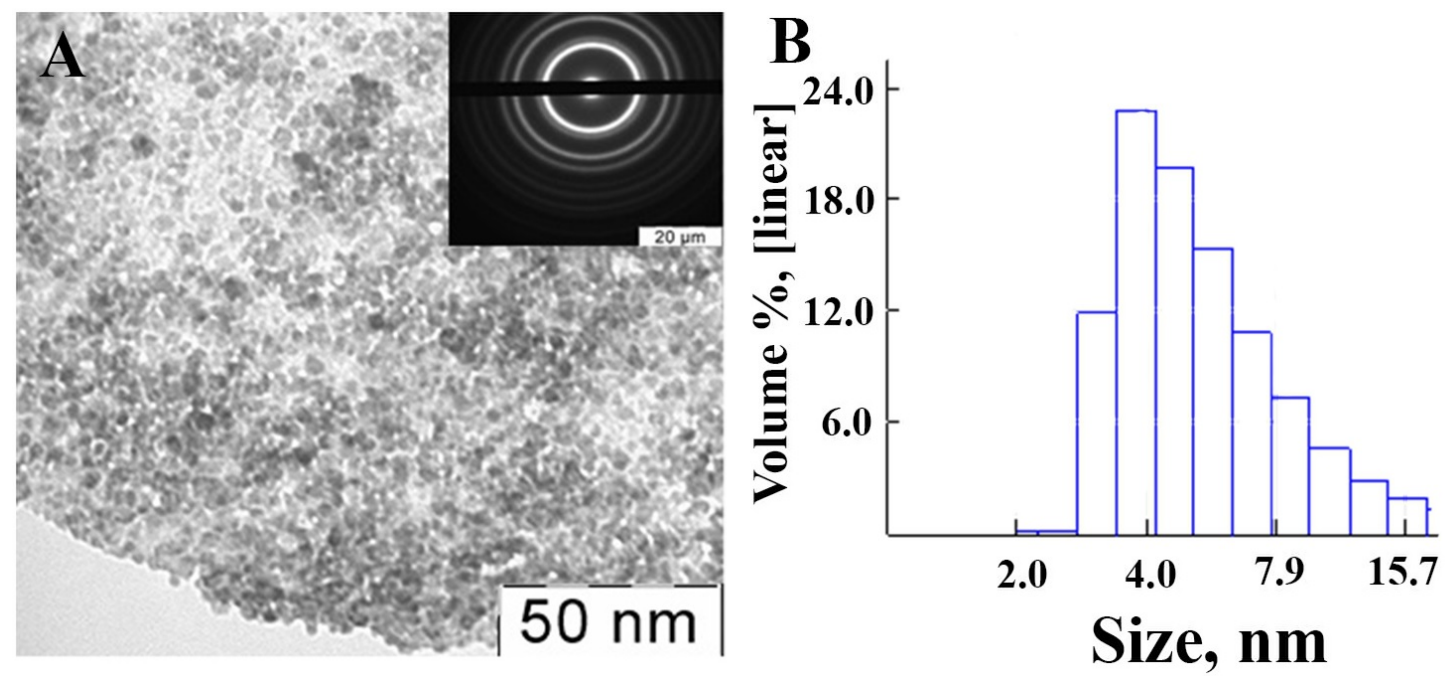

FIG. 2. The transmission electron microscopy image (A), hydrodynamic radii distribution (B) of citrate-stabilized cerium(IV) oxide nanoparticles

Oppositely, irradiation of regenerating planarians with green LED light slowed down the head blastema growth in comparison with the control group which is in accordance with our previous results [26]. The effect of the retard in regeneration depended on the radiation dose. Thus, after $96 \mathrm{~h}$ of regeneration, the size of the blastema for planarians irradiated for 10 min was less than in the control group by $16.6 \%$, while for planarians irradiated for 25 min, the size of blastema was less by $25.3 \%$ against the controls. In the subsequent observation periods, the inhibitory effect of exposure to the green LED light remained, while the difference from the control group became lower. In irradiated planarians, after $96 \mathrm{~h}$ of regeneration, the growth slowed down by $13.3 \%$ and $17.2 \%$ (for 10 and 25 min exposure, respectively). After $120 \mathrm{~h}$ of regeneration, the growth rate decreased by $10.8 \%$ and $12.8 \%$, respectively.

\section{Control}

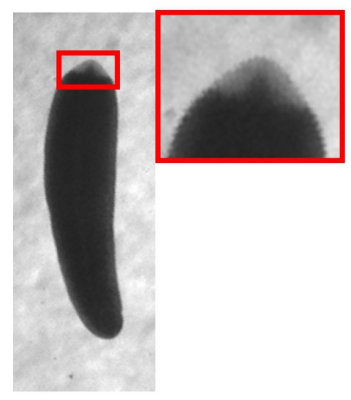

$\mathrm{CeO}_{2}\left(10^{-11} \mathrm{M}\right)$

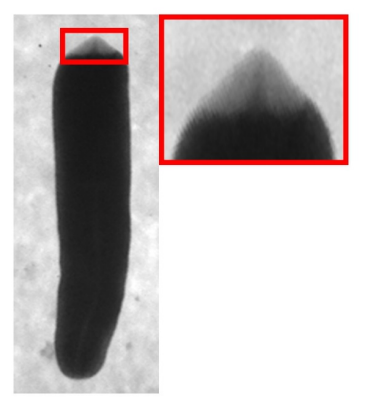

LED 25 min

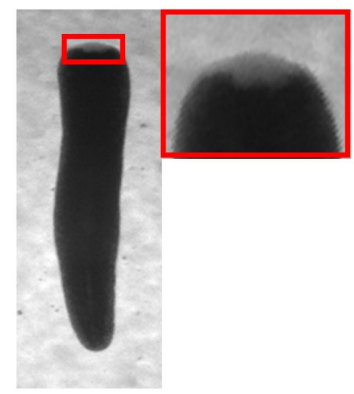

$\mathrm{CeO}_{2}\left(10^{-11} \mathrm{M}\right)$ + LED 25 min

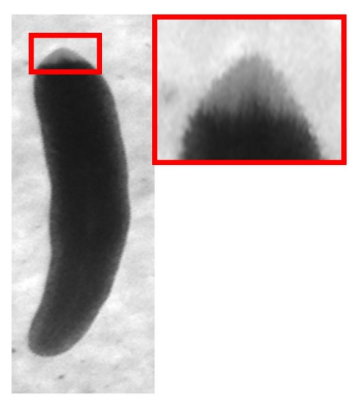

FIG. 3. Micrographs of regenerating planarians after irradiation with green LED source for $25 \mathrm{~min}$ and incubation with $\mathrm{CeO}_{2}$ nanoparticles for $72 \mathrm{~h}$

Illumination with green light inhibited the regeneration of planaria, meanwhile the presence of $\mathrm{CeO}_{2}$ nanoparticles stimulated planaria regeneration. When the animals were exposed to green light for $10 \mathrm{~min}$, the subsequent $72 \mathrm{~h}$ incubation with $\mathrm{CeO}_{2}$ nanoparticles resulted in the higher regeneration rate (17\%) than in the control. The similar difference in $\Delta R$ values (19.4\%) was observed between planarians irradiated with green light for 25 min with subsequent incubation for $72 \mathrm{~h}$ in the presence of $\mathrm{CeO}_{2}$ nanoparticles and the controls.

Further, after 96 and $120 \mathrm{~h}$ of regeneration, a decrease in the difference between the regeneration indices for irradiated and untreated planarians was observed, but the stimulating effect of ceria nanoparticles was still significant. Particularly, the animals irradiated with green light for $10 \mathrm{~min}$ with subsequent regeneration for 96 and $120 \mathrm{~h}$ in the presence of $\mathrm{CeO}_{2}$ nanoparticles showed the $\Delta R$ values of $9.7 \%$ and $8.4 \%$, respectively. Similarly, after 25 min of green light irradiation and subsequent regeneration of planarians for 96 and $120 \mathrm{~h}$ in the presence of $\mathrm{CeO}_{2}$ nanoparticles, $\Delta R$ values amounted to $14.5 \%$ and $12.8 \%$, respectively. 


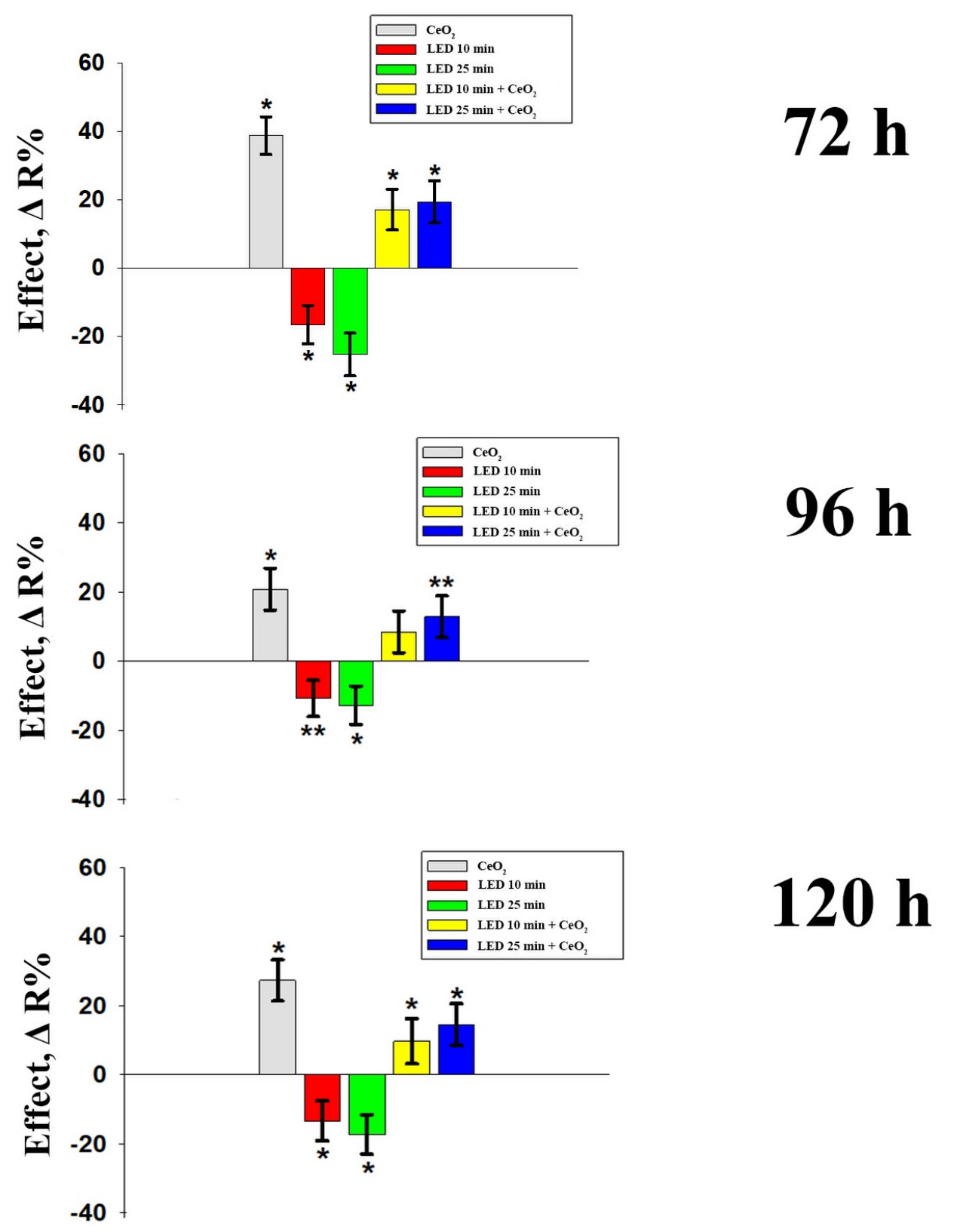

FIG. 4. The effect of cerium(IV) oxide nanoparticles $\left(10^{-11} \mathrm{M}\right)$ and irradiation with green LED light $(525 \mathrm{~nm}$, duration of irradiation -10 and $25 \mathrm{~min}$ ) on planarian blastema regeneration. The effect is shown as a percentage of untreated control after 72,96 and $120 \mathrm{~h}$ of regeneration. ${ }^{*} p<0.001$; ${ }^{* *} p<0.01$

Our experiments have unambiguously shown that ceria nanoparticles, even in very low concentrations $\left(10^{-11} \mathrm{M}\right)$, effectively neutralize the negative effect of green light irradiation on the regeneration processes in planarians. In our opinion, this effect cannot be associated with the photoprotective property of ceria nanoparticles [27] due to low concentration of $\mathrm{CeO}_{2}$ in the aqueous medium. Moreover, being a wide band-gap semiconductor (band gap of $3.2 \mathrm{eV}$ [28]), $\mathrm{CeO}_{2}$ is unable to absorb green light. Thus, the effect is connected with the molecular mechanisms of ceria interaction with biological systems.

Upon damage to the planarian body, stem cells (neoblasts) begin to migrate actively into the area of injury where a burst of ROS levels occurred. According to our recent results, $\mathrm{CeO}_{2}$ nanoparticles greatly promote the mitotic activity of the neoblasts in decapitated planarians [9] probably due to the modulation of ROS level in the apical layer of the blastema. 
The main mechanism of the reaction of biological systems to visible light irradiation is considered to be due to the absorption of photons by certain photoacceptor molecules (photoreceptors or chromophores) [29-31]. Planarians have photo- and chemoreceptors not only in the area of their eye-spots (ocelli), but also throughout the body being able to respond the external light stimuli even upon decapitation. Opsins are G-protein-coupled photoreceptors responsible for ocular light detection in all animals. Opsins are typically located within either rhabdomeric or ciliary photoreceptor cells, activating r-opsin or c-opsin signal transduction cascades, respectively [32]. C-opsins initiate a pathway that closes cyclic-nucleotide-gated (CNG) ion channels [33], whereas r-opsins are associated with the opening of transient receptor potential cation (TRPC) channels [34]. In planarians, the homologs of CNG and TRPC channels are transient receptor potential cation (TRPA1) channels, which are evolutionarily conserved detectors of reactive oxygen species [35]. Therefore, we can assume that through ROS level regulation by cerium oxide nanoparticles, it is possible to indirectly influence biochemical processes governed by photosensitive opsins.

Cerium oxide nanoparticles, as shown earlier, are able to influence the signaling pathways of the cell through the regulation of the redox status of the cell [36]. $\mathrm{CeO}_{2}$ nanoparticles can interact with many biomolecules triggering a cascade of cellular activities [37]. $\mathrm{CeO}_{2}$ nanoparticles-targeted signaling pathways can induce a variety of cellular responses dependent or independent from free radical scavenging. Thus, it can be concluded that the mechanisms of the biological activity of $\mathrm{CeO}_{2}$ nanoparticles are mostly associated with their effect on intracellular signaling pathways. However, specific pathways of signal transduction upon exposure to green light in the presence of $\mathrm{CeO}_{2}$ nanoparticles require additional research.

\section{Conclusions}

Ultra-small citrate-stabilized cerium oxide nanoparticles do not cause toxic effects on planarians and are able to stimulate the mitotic activity of their stem cells during blastema growth. Exposure to green light for 15 and 25 min significantly inhibits the process of planarian regeneration, which is most likely associated with down-regulated expression of genes associated with wound healing. At the same time, cerium oxide nanoparticles, even in nanomolar concentrations, are able to neutralize the negative effects of green light on the regeneration processes in planarians, increasing the rate of regeneration and increasing the mitotic index.

\section{Acknowledgements}

This work was supported by the Russian Science Foundation, project no. 19-13-00416. This research was partly performed using the equipment of the JRC PMR IGIC RAS.

\section{References}

[1] Seminko V., Maksimchuk P., Grygorova G., Okrushko E., Avrunin O., Semenets V., Malyukin Y. Mechanism and Dynamics of Fast Redox Cycling in Cerium Oxide Nanoparticles at High Oxidant Concentration. J. Phys. Chem. C, 2021, 125(8), P. 4743-4749.

[2] Malyukin Y., Maksimchuk P., Seminko V., Okrushko E., Spivak N. Limitations of Self-Regenerative Antioxidant Ability of Nanoceria Imposed by Oxygen Diffusion. J. Phys. Chem. C, 2018, 122(28), P. 16406-1641.

[3] Wang Z.-Q., Zhang M.-J., Hu X.-B., et al. $\mathrm{CeO}_{2-x}$ quantum dots with massive oxygen vacancies as efficient catalysts for the synthesis of dimethyl carbonate. Chem. Commun., 2020, 56, P. 403-406.

[4] Rink, J.C. Stem cell systems and regeneration in planaria. Dev. Genes Evol., 2013, 223, P. 67-84.

[5] Reddien P.W., Alvarado A.S. Fundamentals of Planarian Regeneration. Annual Review of Cell and Developmental Biology, 2004, 20, P. 72575 .

[6] Cutie S., Hoang A.T., Payumo A.Y., Huang G.N. Unconventional Functions of Muscles in Planarian Regeneration. Dev. Cell, 2017, 43(6), P. 657-658.

[7] Reddien P.W. Specialized progenitors and regeneration. Development, 2013, 140(5), P. 951-957.

[8] Pagán O.R. Planaria: an animal model that integrates development, regeneration and pharmacology. Int. J. Dev. Biol., 2017, 61(8-9), P. 519529

[9] Ermakov A., Popov A., Ermakova O., et al. The first inorganic mitogens: Cerium oxide and cerium fluoride nanoparticles stimulate planarian regeneration via neoblastic activation. Materials Science and Engineering: C, 2019, 104, P. 109924.

[10] Popov A.L., Shcherbakov A.B., Zholobak N.M. et al. Cerium dioxide nanoparticles as third-generation enzymes (Nanozymes). Nanosystems: Physics, Chemistry, Mathematics, 2017, 8(6), P. 760-781.

[11] Popova N.R., Andreeva V.V., Khohlov N.V., Popov A.L., Ivanov V.K. Fabrication of $\mathrm{CeO}_{2}$ nanoparticles embedded in polysaccharide hydrogel and their application in skin wound healing. Nanosystems: Physics, Chemistry, Mathematics, 2020, 11(1), P. $99-109$.

[12] Baldim V., Bedioui F., Mignet N., Margaill I., Berret J.-F. The enzyme-like catalytic activity of cerium oxide nanoparticles and its dependency on $\mathrm{Ce}^{3+}$ surface area concentration. Nanoscale, 2018, 10, P. 6971-6980.

[13] Singh R., Singh S. Redox-dependent catalase mimetic cerium oxide-based nanozyme protect human hepatic cells from 3-AT induced acatalasemia. Colloids and Surfaces B: Biointerfaces, 2019, 175, P. 625-635.

[14] Li Y., Hou X., Yang C. et al. Photoprotection of cerium oxide nanoparticles against UVA radiation-induced senescence of human skin fibroblasts due to their antioxidant properties. Scientific Reports, 2019, 9, P. 2595.

[15] Caputo F., De Nicola M., Sienkiewicz A. et al. Cerium oxide nanoparticles, combining antioxidant and UV shielding properties, prevent UV-induced cell damage and mutagenesis. Nanoscale, 2015, 7, P. 15643-15656. 
[16] Arya A., Gangwar A., Singh S. K., Bhargava K. Polyethylene glycol functionalized cerium oxide nanoparticle confer protection against UV-induced oxidative damage in skin: evidences for a new class of UV filter. Nano Ex., 2020, 1, P. 010038.

[17] Miri A., Birjandi S.A., Sarani M. Survey of cytotoxic and UV protection effects of biosynthesized cerium oxide nanoparticles. J. Biochem. Mol. Toxicol. 2020, 34(6), P. e22475.

[18] Yang J., Xu Z.-P., Huang Y. et al. ATM and ATR: Sensing DNA damage. World J Gastroenterol., 2004, 10(2), P. 155-160.

[19] Caputo F., Giovanetti A., Corsi F. et al. Cerium oxide nanoparticles re-establish cell integrity checkpoints and apoptosis competence in irradiated HaCat cells via novel redox-independent activity front. Pharmacol, 2018, 9, P. 1183.

[20] Popov A. L., Zaichkina S. I., Popova N. R. et al. Radioprotective effects of ultra-small citrate-stabilized cerium oxide nanoparticles. RSC Advances, 2016, 6, P. 106141-106149.

[21] Briggs A., Corde S., Oktaria S. et al. Cerium oxide nanoparticles: influence of the high-Z component revealed on radioresistant 9L cell survival under X-ray irradiation. Nanomedicine: Nanotechnology, Biology and Medicine, 2013, 9(7), P. 1098-1105.

[22] Shinpaugh J.L. et al Protection and sensitization of normal and tumor cells to proton radiation by cerium oxide nanoparticles. J. Phys.: Conf. Ser., 2015, 635, P. 032032

[23] Ermakov A.M., Ermakova O.N., Maevsky E.I. A role of some intracellular signaling cascades in planarian regeneration activated under irradiation with low-temperature argon plasma. Biophysics, 2014, 59, P. 453-457.

[24] Ivanova O.S., Shekunova T.O., Ivanov V.K. et al. One-step synthesis of colloidal solutions of cerium dioxide for biomedical applications. Doklady Chemistry, 2011, 437(2), P. 103-106.

[25] Popov A., Popova, N., Gould D., Shcherbakov A.; Sukhorukov G.; Ivanov V. Ceria nanoparticles-decorated microcapsules as a smart drug delivery/protective system: Protection of encapsulated P. pyralis luciferase. ACS Appl. Mater. Interfaces, 2018, 10(17), P. 14367-14377.

[26] Ermakov A.M., Ermakova O.N., Popov A.L., Manokhin A.A., Ivanov V.K. Opposite effects of low intensity light of different wavelengths on the planarian regeneration rate. Journal of Photochemistry and Photobiology, B: Biology, 2020, 202, P. 111714.

[27] Ribeiro F.M., de Oliveira M.M., Singh S., Sakthivel T.S., Neal C.J., Seal S., Ueda-Nakamura T., Lautenschlager S., Nakamura C. Ceria Nanoparticles Decrease UVA-Induced Fibroblast Death Through Cell Redox Regulation Leading to Cell Survival, Migration and Proliferation. Front. Bioeng. Biotechnol., 2020, 8, P. 577557.

[28] Shcherbakov A.B., Zholobak N.M., Ivanov V.K. Biological, biomedical and pharmaceutical applications of cerium oxide. In Cerium Oxide $\left(\mathrm{CeO}_{2}\right)$ : Synthesis, Properties and Applications. Elsevier. Amsterdam. 2020, 402 p.

[29] de Freitas L.F., Hamblin M.R. Proposed Mechanisms of Photobiomodulation or Low-Level Light Therapy. IEEE J. Sel. Top. Quantum. Electron., 2016, 22(3), P. 7000417.

[30] Passarell S., Karub T. Absorption of monochromatic and narrow band radiation in the visible and near IR by both mitochondrial and nonmitochondrial photoacceptors results in photobiomodulation. Journal of Photochemistry and Photobiology B, 2014, 140, P. 344-358.

[31] Arendt D. Evolution of eyes and photoreceptor cell types. Int J Dev Biol., 2003, 47(7-8), P. 563-571.

[32] Shettigar N., Joshi A., Dalmeida R., Gopalkrishna R., Chakravarthy A., Patnaik S., Mathew M., Palakodeti D., Gulyani A. Hierarchies in light sensing and dynamic interactions between ocular and extraocular sensory networks in a flatworm. Science Advances, 2017, 3(7), P. e1603025.

[33] Kaupp B. and Seifert R., Cyclic nucleotide-gated ion channels. Physiol Rev., 2002, 82(3), P. 769-824.

[34] Katz B., Payne R., Minke B. TRP Channels in Vision. In: Neurobiology of TRP Channels. CRC Press/Taylor \& Francis. Boca Raton, 2017, $347 \mathrm{p}$.

[35] Birkholz T.R., Beane W.S. The planarian TRPA1 homolog mediates extraocular behavioral responses to near ultraviolet light. Journal of Experimental Biology, 2017, 152298.

[36] Weng Q. et al., Catalytic activity tunable ceria nanoparticles prevent chemotherapy-induced acute kidney injury without interference with chemotherapeutics. Nat. Commun., 2021, 12, P. 1436.

[37] Pesaraklou A., Matin M. M. Cerium oxide nanoparticles and their importance in cell signaling pathways for predicting cellular behavior. Nanomedicine, 2020, 15, P. 17. 